\title{
Aspectos éticos en la investigación cualitativa
}

\author{
Martha Lilia Parra Domínguez,* Isías Iván Briceño Rodríguez**
}

\begin{abstract}
RESUMEN
Como estudio de la moral, la ética es, ante todo, una filosofía práctica, cuya tarea no es precisamente resolver los conflictos, sino plantearlos. La calidad de la investigación cualitativa es un aspecto fundamental que los investigadores permanentemente deben garantizar y que los profesionales de los servicios de salud necesitan evaluar antes de utilizar los resultados de los estudios. Cuando hablamos de calidad de la investigación, aludimos al rigor metodológico con que ha sido diseñada y desarrollada y a la confianza que, como consecuencia de ello, podemos tener en la veracidad de los resultados conseguidos. Por tanto, la idea de calidad de la investigación se asocia, en general, a la credibilidad del trabajo desarrollado. Los estándares que permiten evaluar el rigor y carácter científico de los estudios cualitativos y sobre los cuales hay acuerdo parcial son la credibilidad, la auditabilidad o confirmabilidad y la transferibilidad o aplicabilidad.
\end{abstract}

Palabras clave: Ética, rigor científico, investigación cualitativa.

\section{Ethical issues in qualitative research}

\begin{abstract}
As a study of morality, ethics is primarily practical philosophy, which task is not precisely to resolve conflicts, but to raise them. The respectability of qualitative research is a fundamental aspect that researchers must guarantee permanently and that health professionals should assess before using the findings of the studies. When we say quality of research we refer to the methodological rigor with which it has been designed and developed, and, as a result of this, to how we can trust the accuracy of the results achieved. In general, the idea of quality of research is therefore associated with the credibility of the work performed. Quality standards to assess the rigor and scientific character of qualitative studies and for which there is partial agreement are: credibility, confirmability or auditability, and transferability or applicability.
\end{abstract}

Key words: Ethics, scientific rigor, qualitative research.

\section{INTRODUCCIÓN}

$\mathrm{E}$ ejercicio de la investigación científica y el uso del conocimiento producido por la ciencia demandan conductas éticas en el investigador y el maestro. $\mathrm{La}$ conducta no ética no tiene lugar en la práctica científica de ningún tipo. Debe ser señalada y erradicada. Aquel que con intereses particulares desprecia la ética en una investigación, corrompe a la ciencia, sus productos, y se corrompe a sí mismo.

La investigación cualitativa comparte muchos aspectos éticos con la investigación convencional. Así, los aspectos éticos que son aplicables a la ciencia en general, son aplicables a la investigación cualitativa. La práctica científica

*Doctora en Ciencias de Enfermería. Profesora principal en la Facultad de Enfermería y Obstetricia de la Universidad Juárez del Estado de Durango. **Maestro en Ciencias de Enfermería. Profesor en la Facultad de Enfermería y Obstetricia de la Universidad Juárez del Estado de Durango.

Correspondencia: Martha Lilia Parra Domínguez. Email: mlpd73@hotmail.com

Este artículo puede ser consultado en versión completa en http://www.medigraphic.com/enfermerianeurologica 
como práctica de la libertad es igual cuando realizamos investigación cualitativa. Sin embargo, los problemas, los métodos, la comunicación y la divulgación de la investigación cualitativa plantean algunos conflictos adicionales. La investigación cualitativa reconoce la subjetividad de los individuos como parte constitutiva de su proceso indagador. Ello implica que las ideologías, las identidades, los juicios, los prejuicios y todos los elementos de la cultura impregnan los propósitos, el problema, el objeto de estudio, los métodos e instrumentos; forman parte de la selección de los recursos y los mecanismos empleados para hacer la presentación y divulgación de los resultados e interpretaciones del estudio.

La investigación cualitativa tiene desafíos adicionales. En las ciencias, indaga en la condición humana, construyendo el conocimiento mientras acoge la complejidad, la ambigüedad, la flexibilidad, la singularidad, la pluralidad, lo contingente, lo histórico, lo contradictorio y lo afectivo, entre otras circunstancias propias de la subjetividad del ser humano y su carácter social. Estas condiciones son características del objeto de estudio a la luz del enfoque cualitativo y, al mismo tiempo, son también valores cultivados durante la investigación, porque, en buena medida, la riqueza de la investigación cualitativa depende de qué tan bien hemos captado y descrito dichas particularidades en la búsqueda de los significados.

A partir del enfoque cualitativo, aceptamos que el objeto de la investigación es un sujeto interactivo, motivado e intencional, que asume una posición frente a las tareas que enfrenta. Por esta razón, no se puede ignorar que la investigación es un proceso de comunicación entre investigador e investigado, un diálogo que toma diferentes formas. ${ }^{1}$

Particularmente en la investigación cualitativa, es necesario incluir ciudadanos con capacidad y disposición de reflexión y comunicación para comprender los valores sociales, las prioridades, la vulnerabilidad y las inquietudes de los sujetos potenciales del estudio. La pluralidad es otra condición deseable en los grupos evaluadores, que debe ser atendida en la investigación cualitativa e incorporada en el método que se utiliza para construir los juicios éticos. ${ }^{2}$

En los últimos 50 años, el desarrollo de la ética en la indagación científica estuvo preferentemente dirigido a los estudios con pacientes en el campo de la salud-enfermedad. Las fuentes principales de orientación ética sobre la realización de investigaciones clínicas han sido el Código de Nuremberg, la Declaración de Helsinki, el Informe Belmont y las normas del Consejo para la Organización Internacional de Ciencias Médicas. El momento alcanzado por la bioética como estudio de lo moral orientado a la salud es sumamente fuerte y amplio, y ha sido un estímulo vigoroso para el fortalecimiento de la ética.
Poco a poco las ciencias de la salud han ido recuperando, no sin dificultad, algo que ha estado escrito desde siempre en la forma de observar y transformar la realidad: la vivencia subjetiva del proceso de enfermar y la condición probable, sin certeza, de todo intento de intervenir en dichos procesos. ${ }^{3}$ En consecuencia, los métodos cualitativos han vuelto a ocupar la posición que les correspondía, por su naturaleza, en el marco de la investigación en salud. Además, la investigación cualitativa puede contribuir a evaluar la calidad del cuidado de la salud y a complementar la investigación cuantitativa.

Sin embargo, el retorno de los métodos cualitativos también tiene sus riesgos. Uno muy importante, que tiene que ver precisamente con el hecho de quedar relegados en décadas pasadas en el campo de la biomedicina, estriba en pasar por alto la sólida reflexión desarrollada en torno a las responsabilidades éticas de los que investigan con seres humanos. Esta ponderación ha estado vinculada sobre todo a los métodos cuantitativos. Y es que a veces parece, incluso a los mismos "cualitivistas", que el método cualitativo, al etiquetarse de tecnología blanda o humanista, al estar lejos de la dureza de la estadística, del riesgo de daño físico y de la rigidez del ensayo aleatorizado, está ya de por sí justificado y no plantea problemas éticos que precisan de la evaluación y supervisión. ${ }^{4}$ Algunos de los cuestionamientos que se hace el estudioso durante toda la investigación cualitativa son: ¿cómo garantizar el rigor del trabajo científico?, ¿cómo otros investigadores juzgarán el rigor científico de la investigación realizada? Para dar respuesta a estas interrogantes, se debe tomar en cuenta que la investigación cualitativa se caracteriza por ver las cosas desde el punto de vista de las personas que están siendo estudiadas. De esta manera, el papel de los investigadores es entender e interpretar qué está sucediendo; esto es una tarea difícil: por un lado, porque los científicos no pueden abstraerse totalmente de su propia historia, sus creencias y su personalidad; y por otro, por la complejidad de los fenómenos humanos.

Retomando el análisis sobre lo que implica el rigor metodológico, algunos investigadores cualitativos afirman que los cánones o estándares con que se juzgan los estudios cuantitativos son totalmente inapropiados para evaluar el rigor metodológico de los estudios cualitativos. ${ }^{5}$ El fundamento para esta apreciación es que los abordajes cualitativos y cuantitativos tienen diferentes raíces ontológicas y epistemológicas, que deben comprenderse, respetarse y mantenerse durante todo el proceso investigativo. Los científicos cualitativos concuerdan en ese principio rector y, por tanto, estos criterios o estándares de evaluación deben ser coherentes con los propósitos, fines y bases filosóficas del paradigma cualitativo. Los cánones o estándares usuales en la investigación cuantitativa incluyen dos elementos: la validez y la confiabilidad. Extrapolar estos criterios 
a la investigación cualitativa es contraproducente, pues se violan los propósitos, los objetivos y la integridad del abordaje cualitativo. Esto no quiere decir que la investigación cualitativa se exonere del rigor metodológico que debe tener todo proceso de búsqueda científica; se refiere a que los criterios usados para juzgar el mérito del trabajo cualitativo también deben ser explícitos, utilizando la credibilidad, la auditabilidad y la transferibilidad para evaluar la calidad científica. ${ }^{6}$

La credibilidad se logra cuando el investigador, a través de sus observaciones y conversaciones prolongadas con los participantes en el estudio, recolecta información que produce hallazgos reconocidos por los informantes como una verdadera aproximación a lo que ellos piensan y sienten. Así, la credibilidad se refiere a cómo los resultados de una investigación son verdaderos para las personas que fueron estudiadas y para otros individuos que han experimentado o estado en contacto con el fenómeno investigado. La credibilidad (validez interna), aumenta la probabilidad de que los datos hallados sean creíbles; se pueden contrastar los resultados con las fuentes mediante la observación persistente, los diarios de experiencias, las encuestas, los análisis de documentos, la discusión grupal y la triangulación; puede ser una negociación inicial y durante todo el proceso. En el carácter científico es una obligación ética seguir un método científicamente correcto que asegure su validez interna. En el caso de la investigación cualitativa, se exige que el diseño metodológico sea adecuado para los objetivos que se propone, que seleccione oportunamente los contextos y los informantes, y que interprete acertadamente los resultados. ${ }^{7}$ La credibilidad puede ser alcanzada cuando el investigador vuelve a los informantes durante la recolección de información, lo que permite confirmar los hallazgos y revisar algunos datos particulares. En este sentido, la mayoría de los informantes son capaces de corregir los errores de interpretación de los hechos y, para ello, se ocupan de dar más ejemplos que ayudan a clarificar las interpretaciones del investigador.

El segundo criterio es la auditabilidad, llamada también confirmabilidad. Éste se describe como la habilidad de otro investigador de seguir la pista o ruta de lo que el científico original ha hecho. Para ello es necesario un registro y documentación completa de las decisiones e ideas que el estudioso haya tenido en relación con el trabajo. Esta estrategia permite que otro investigador examine los datos y pueda llegar a las conclusiones iguales o similares a las del científico original, siempre y cuando tengan perspectivas similares. ${ }^{8}$

La transferibilidad o aplicabilidad es el tercer criterio que se toma en cuenta para el rigor metodológico, y se refiere a la posibilidad de extender los resultados del estudio a otras poblaciones. En la investigación cualitativa, la audiencia o el lector del informe son los que determinan si pueden transferir los hallazgos a un contexto diferente del análisis. Para ello es necesario que se describan densamente el lugar y las características de las personas donde el fenómeno fue observado. Por tanto, el grado de transferibilidad es una función directa de la similitud entre los contextos.

Morse $^{9}$ plantea que los conceptos o constructos de confiabilidad y validez deben retomarse en una investigación cualitativa como estándares de rigor científico, por tres razones:

1. La validez y la confiabilidad son estándares de rigor científico independientemente de los paradigmas que orienten la investigación, porque el objetivo fundamental de toda investigación es encontrar resultados plausibles y creíbles.

2. Rehusarse a utilizar los estándares de validez y credibilidad conduce a la marginalización de la investigación cualitativa del paradigma científico predominante; es decir, se está contribuyendo a fortalecer la noción de que la investigación cualitativa es inválida, no confiable, falta de rigor y, por tanto, no científica.

3. Los criterios de credibilidad, auditabilidad y transferibilidad propuestos por Guba y Lincoln enfatizan la evaluación del rigor científico al finalizar la investigación, lo cual tiene el riesgo de que el investigador no identifique las amenazas contra la validez y confiabilidad del estudio, y las corrija durante el mismo.

Morse propone que es el momento de considerar la importancia de que el científico use estrategias de verificación durante el proceso de investigación; de esta manera, la validez y la confiabilidad se obtienen activamente y no son aspectos evaluados por pares externos al finalizar el proyecto investigativo. Es decir, las estrategias de verificación deben ser parte del trabajo cualitativo. Las estrategias de verificación que propone esta autora son:

1. La creatividad, sensibilidad, flexibilidad y habilidad del investigador para "responder" a lo que sucede durante el estudio.

2. La coherencia metodológica, es decir, la congruencia entre la pregunta de investigación y los componentes del método usado. La interdependencia del trabajo cualitativo demanda que la pregunta concuerde con el método, con la información recabada y el análisis de ésta.

3. Muestreo apropiado: seleccionar a los participantes que mejor representen o tengan conocimiento del fenómeno a investigar. Esto garantiza una saturación efectiva y eficiente de las categorías con información de calidad óptima y mínimo desperdicio. 
4. Recolección y análisis de información concurrentes para lograr la interacción entre lo que se desconoce y lo que se necesita conocer. Esta interacción es esencial para lograr la validez y la confiabilidad.

5. Saturación de la información: se alcanza cuando hay redundancia en la información, esto es, cuando el investigador obtiene la misma información o similar, pues los informantes no indican algo diferente de lo ya dicho.

Otro aspecto importante que se debe tener muy en cuanta para sustentar la ética de todo estudio es el consentimiento informado, uno de los pilares básicos de la ética de la investigación moderna, que, además de ser un principio ético, se ha convertido en una norma jurídica. Todas las leyes que regulan la investigación en el mundo lo marcan como obligación clave a respetar por cualquier estudioso. ${ }^{10}$

Para finalizar, se resaltan dos aspectos centrales en el abordaje cualitativo:

1. Para que la investigación cualitativa tenga calidad, es necesario que los investigadores cumplan con los cánones del rigor metodológico descrito.

2. Además, no sólo se debe generar conocimiento, sino contribuir a la solución de los problemas relevantes para los seres humanos.

\section{CONCLUSIÓN}

Las consideraciones éticas no constituyen un tema aparte de los métodos en la investigación cualitativa, con los cuales se impregnan y confunden. La discusión de la ética en la investigación cualitativa debe ser ampliada con la participación de académicos e investigadores en una convicción pluralista, en instituciones y organizaciones que hacen investigaciones; así se podría contribuir a un aumento en la calidad de la investigación cualitativa.

\section{BIBLIOGRAFÍA}

1. González R. Investigación cualitativa en psicología. Rumbos y desafíos México, DF: Thompson Editores Internacional; 2000. pp. 35-39.

2. Emanuel E. ¿Qué hace que la investigación sea ética? Siete requisitos básicos. En: Pellegrini A, Macklin R, editores. Investigación en sujetos humanos: experiencia internacional. Serie Publicaciones. Santiago de Chile: Programa Regional de Bioética OPS/OMS; 1997. pp. 33-46.

3. Conde GF. Encuentros y desencuentros entre la perspectiva cualitativa en la historia de la medicina. Rev Esp Salud Pública. 2002; 76: 395-408.

4. Directiva 2001/20/CE del Parlamento Europeo y del Consejo, de 4 de abril de 2001, relativa a la aproximación de las disposiciones legales, reglamentarias y administrativas de los estados miembros sobre la aplicación de buenas prácticas clínicas en la realización de ensayos clínicos de medicamentos de uso humano. Diario Oficial de la Comunidades Europeas. L-121 (01-05-2001)

5. Streubert H, Carpenter D. Qualitative research in nursing. Philadelphia: JB Lippincott Company; 1995.

6. Lincoln YS, Guba EG. Naturalistic Inquiry. Beverly Hills: Sage Publications; 1985.

7. Pla M. El rigor en la investigación cualitativa. Aten Primaria. 1999; 24 295-300.

8. Guba EG, Lincoln YS. Effective evaluation: improving the usefulness of evaluation result through responsive and naturalistic approaches. San Francisco: Jossey-Bass; 1981.

9. Morse J, Barret M, Mayan M et al. Verification strategies for establishing reliability and validity in qualitative research. Int J Qual Meth [Internet]. 2002: (2): [19 pp.]. Disponible en: http://www.ualberta.ca/ijqm

10. Instrumento de ratificación del Convenio para la protección de los derechos humanos y la dignidad del ser humano con respecto a las aplicaciones de la biología y la medicina. (Convenio relativo a los derechos humanos y la biomedicina.) Oviedo, 4 de abril de 1997. BOE. No. 251 (20-10-1999). 